\title{
UN CASO DE POSIBLE INFLUENCIA MAYA EN EL ESPAÑOL MEXICANO
}

Muy recientemente he estudiado las realizaciones polimórticas de varios fonemas que recogimos durante nuestras encuestas dialectales en una región del sureste de México integrada por los estados de Tabasco, Campeche y Yucatán ${ }^{1}$. Advertía entonces que uno de los casos en que el polimorfismo ${ }^{2}$ se mostraba más intenso era el relativo a la articulación de la $[-r]$ final de palabra o de sílaba ${ }^{3}$. Una de las variantes articulatorias que más llamó nuestra atención fue una $r$ retrofleja o cacuminal ${ }^{4}$, que no habíamos hallado hasta entonces en ninguna de las regiones mexicanas investigadas. Este alófono se recogió en cinco de las seis poblaciones entonces estudiadas: Tizimín y Ticul (Yucatán), Champotón y Mamantel (Campeche), y en Emiliano Zapata (Tabasco). Únicamente en las encuestas hechas en Villahermosa (Tabasco) no lo recogimos.

Antes de continuar, juzgo conveniente dar algunas breves noticias sobre nuestro método de investigación dialectal. Todos nuestros viajes de estudio forman parte de un amplio proyecto encaminado a determinar cuáles son las actuales regiones lingüísticas de Méxi$\operatorname{co}^{5}$. En cada una de las localidades visitadas hacemos dos clases de

1 Cf. "Algunos casos de polimorfismo fonético en México", que se publicará en el Homenaje a D. Vicente García de Diego organizado por el C.S.I.C. de Madrid.

2 En estos trabajos he atendido no sólo al polimorfismo puro, individual (idiolectal), sino también al dialectal, en la modalidad que Allières ha denominado "polimorfismo de realizaciones indiferentes" (cf. JacQues Albmìres, "Un exemple de polymorphisme phonétique: le polimorphisme de l'-s implosif en gascon garonnais", $A F L T, 1954$, num. 1, 70-103) .

3 Los otros fonemas que allí estudiaba eran $/ \mathrm{f} / \mathrm{s} / \mathrm{s} / \mathrm{y} / \mathrm{y} /$ en posición intervocálica.

4 Para evitar problemas de imprenta en la transcripción de este alófono retroflejo, lo representaré por $[\mathrm{R}]$. Esta articulación ha aparecido también como realización de $/ \overline{\mathrm{r}} /$, en casos como [péro] o [Róto], pero es más frecuente como realización de /r/ implosiva: [kárne], [tarcle], [kalór], etc.

5 Cf. "Las zonas dialectales de México: Proyecto de delimitación", NRFH, 19 (1970), 1-11, y, con mayor amplitud, "Dialectología mexicana y sociolin- 
encuestas: por un lado, entrevistamos a tres o cuatro informantes -de distinto sexo, nivel sociocultural y edad- con un cuestionario preparado para tal fin, en el cual figuran 400 preguntas de carácter fonético y 600 de índole gramatical o léxica ${ }^{\theta}$; por otro lado, grabamos en cintas magnetofónicas conversaciones libres -de media hora de duración por lo general- sostenidas por el investigador con el informante o, a veces, por dos informadores entre sí. La sistematicidad de los datos obtenidos mediante el primer procedimiento queda completada con la mayor seguridad -por su espontaneidad- de los datos reunidos en las grabaciones magnetofónicas. Los investigadores trabajan en equipo: en primer lugar, son dos normalmente los investigadores que cubren cada viaje, distribuyéndose el trabajo y cordinando las actividades; en segundo lugar, el análisis y procesamiento posterior de los datos reunidos se hace conjuntamente. Tratamos de evitar con ello posibles errores de interpretación, sobre todo en lo que respecta a los materiales fonéticos. Los investigadores que hicieron las encuestas en las poblaciones que aquí estudio son: José Moreno de Alba, Antonio Millán, Antonio Alcalá y Gustavo Cantero ${ }^{7}$. Ellos mismos transcribieron después las cintas allí grabadas y sistematizaron los informes consignados en los cuestionarios.

En las cinco poblaciones donde apareció la [R] retrofleja entrevistamos un total de 36 informantes ${ }^{8}$. En quince de ellos, esa extraña articulación aparecía con cierta regularidad, aunque siempre en concurrencia -dado el intenso polimorfismo de su habla-con realizaciones normales (vibrantes o fricativas) o asibiladas (ya sonoras, ya ensordecidas)

En el artículo citado en la nota l, tras consignar la existencia de este sorprendente alófono y su relativa vitalidad en la zona, apuntaba la posibilidad de relacionarlo con la lengua indígena ampliamente hablada todavía en la región: el maya. Esa posibilidad es la que pretendo estudiar aquí. Para hacer el análisis con un margen mayor de seguridad, me ha parecido adecuado relacionar los

güística", en la misma revista, 23 (1974), 1-34. En estas publicaciones se puede hallar información más detallada sobre lo que aquí presento de manera muy esquemática.

6 Cf. J. M. Lofe Blanch, Cuestionario para la delimitación de las zonas dialectales de México, E1 Colegio de México, 1970.

7 'También Raúl Ávila participó en el viaje a Villahermosa, pero en esta población no recogimos - como he indicado- la [R] cacuminal.

8 En Ticul, a ocho; y a siete en cada una de las restantes localidades. De ellos, doce eran mujeres. Por obvias razones, hemos preferido dar prioridad numérica a los informantes de escasa o nula instrucción escolar; así, en este caso, 19 sujetos eran analfabetos o poco menos; 14 poseían una instrucción media; y sólo 3 podrían considerarse cultos. Sus edades oscilaban entre $\operatorname{los} 16$ y $\operatorname{los} 80$ años. 
hechos lingüísticos recogidos en esas cinco localidades con los recopilados en otras poblaciones de las comarcas circundantes o vecinas, así como atender a diversas circunstancias histórico-culturales que pueden arrojar luz sobre el problema.

I. En nuestras encuestas, hemos recogido también esa articulación levemente retrofleja de $/ \mathrm{r} /$ en las siguientes localidades: en Mérida, Valladolid, Felipe Carrillo Puerto y Huimanguillo con notable regularidad y relativamente alta frecuencia ${ }^{9}$; en menor medida la documentamos también en Chetumal y Campeche, y sólo esporádicamente en Pijijiapan y Tapanatepec. No la oímos, en cambio, ni en Tonalá, San Cristóbal las Casas y Comitán (Chiapas), ni en Frontera (Tabasco) y Minatitlán (Veracruz), sin que esto, como es lógico, permita asegurar que la $[R]$ no exista en alguna de estas últimas localidades, siquiera sea en forma esporádica. Pero lo que resulta evidente es que la articulación retrofleja alcanza su mayor vitalidad en la zona norte de la península de

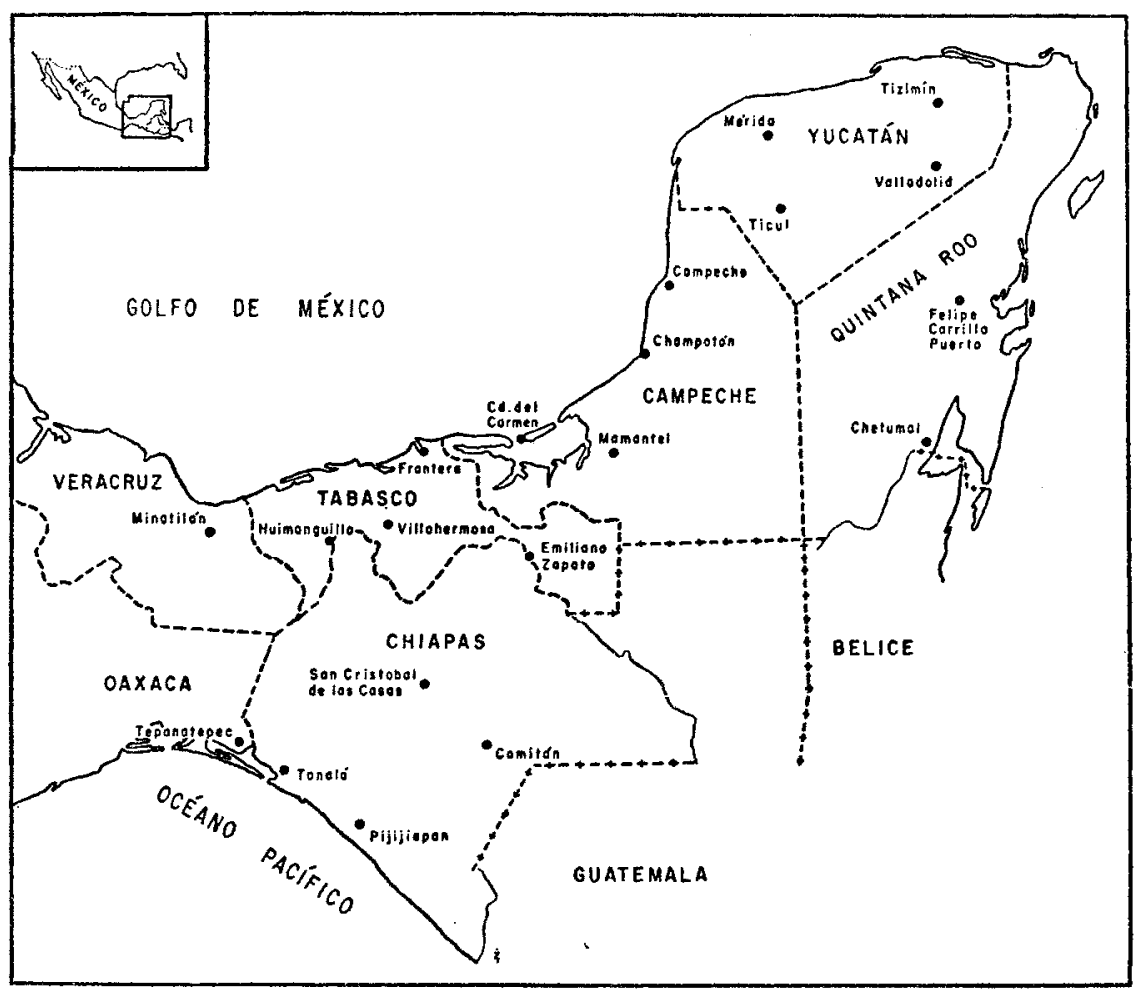

9 Seis de los ocho informantes entrevistados en Mérida y en Valladolid la practicaban más o menos regularmente, asi como cinco de los siete informadores de Felipe Carrillo Puerto, y seis de los siete entrevistados en Huimanguillo, aunque aquí con bajo indice de aparición. 
Yucatán y pierde fuerza conforme se aleja de ese foco yucateco ${ }^{10}$. El área geográfica del fenómeno parece, pues, coincidir con el territorio de base lingüística maya, en especial con las regiones donde el maya sigue siendo lengua comúnmente hablada.

II. Como es sabido, el idioma maya mantiene todavía una situación especial - de privilegio- entre todas las lenguas indígenas mexicanas. Goza de un prestigio socio-cultural de que carecen los restantes idiomas amerindios del país, lo cual le ha permitido mantener una vitalidad extraordinaria frente a la lengua oficial. Y ello, muy especialmente, en el territorio de los actuales estados de Yucatán y Quintana Roo, que ocupan la zona norte y noreste de la península yucateca, es decir, la región extrema de la península, la más alejada del resto del país.

El censo relativo a la situación lingüística de esa zona es sumamente revelador ${ }^{11}$. El número de hablantes de la lengua indígena supera con mucho al promedio del país y aun al de las regiones vecinas. Tanto en Yucatán como en Quintana Roo, más de la mitad de la población habla maya: el $55.5 \%$ y el $54 \%$ respectivamente ${ }^{12}$.

\begin{tabular}{|c|c|c|c|c|c|c|}
\hline & Yucatán & $\begin{array}{l}\text { Quintana } \\
\text { Roo }\end{array}$ & $\begin{array}{c}\text { Campe- } \\
\text { che }\end{array}$ & Tabasco & Veracruz & Guanajuato \\
\hline $\begin{array}{l}\text { Población } \\
\text { total }\end{array}$ & 643,432 & 71,502 & 210,511 & 628,721 & $3.171,856$ & $1.868,869$ \\
\hline Español & $\begin{array}{c}286,162 \\
(44.5 \%)\end{array}$ & $\begin{array}{r}32,973 \\
(46 \%)\end{array}$ & $\begin{array}{c}153,480 \\
(73 \%)\end{array}$ & $\begin{array}{r}594,533 \\
(94.6 \%)\end{array}$ & $\begin{array}{r}2.811,547 \\
(88.6 \%)\end{array}$ & $\begin{array}{c}1.866,597 \\
(99.8 \%)\end{array}$ \\
\hline Bilingües & $\begin{array}{c}300,700 \\
(46.7 \%)\end{array}$ & $\begin{array}{l}30,305 \\
(42.4 \%)\end{array}$ & $\begin{array}{c}51,111 \\
(24.3 \%)\end{array}$ & $\begin{array}{r}31,988 \\
(5 \%)\end{array}$ & $\begin{array}{c}270,686 \\
(8.5 \%)\end{array}$ & $\begin{array}{c}1,858 \\
(0.09 \%)\end{array}$ \\
\hline $\begin{array}{l}\text { Maya o } \\
\text { Leng. in- }\end{array}$ & $\begin{array}{c}56,570 \\
(8.8 \%)\end{array}$ & $\begin{array}{r}8,224 \\
(11.5 \%)\end{array}$ & $\begin{array}{r}5,920 \\
(2.8 \%)\end{array}$ & $\begin{array}{r}2,200 \\
(0.35 \%)\end{array}$ & $(2.8 \%)$ & $(0.02 \%)$ \\
\hline \begin{tabular}{l}
\multicolumn{2}{c}{ digena } \\
Total ha- \\
blantes \\
lengua \\
indígena
\end{tabular} & $\begin{array}{c}357,270 \\
(55.5 \%)\end{array}$ & $\begin{array}{r}38,529 \\
(54 \%)\end{array}$ & $\begin{array}{r}57,031 \\
(27 \%)\end{array}$ & $\begin{array}{c}34,188 \\
(5.5 \%)\end{array}$ & $\begin{array}{c}360,309 \\
(11.3 \%)\end{array}$ & $\begin{array}{c}2,272 \\
(0.12 \%)\end{array}$ \\
\hline
\end{tabular}

10 Aunque mantiene vigor en algunas localidades de la zona meridional, como Emiliano Zapata y Huimanguillo, poblaciones un tanto aisladas y alejadas -tal vez- de la norma "oficial" o standard tabasqueña.

11 Los datos de ese censo, que utilizo a continuación, corresponden al año de 1970. En su aspecto lingüístico, atienden sólo a la población mayor de 5 años.

12 Cierto es que estos datos son de validez relativa, ya que en el censo no se especifica grado de conocimiento de la lengua ni otros detalles de gran interés lingüístico. Por otro lado, y a juzgar por los datos reunidos en censos anteriores, parece apreciarse una paulatina debilitación de la lengua maya 
La situación lingüística de ambos estados es muy diferente de la del resto del país ${ }^{13}$ : la mayor parte de su población es bilingüe $(46.7 \%$ y $42.4 \%)$, y las personas que sólo hablan español no representan siquiera la mitad de la población total $(44.5 \%$ y $46 \%)$, mientras que en los estados vecinos - Campeche y Tabasco- la población exclusivamente hispanohablante asciende al $73 \%$ y al $94.6 \%$ respectivamente, y en un estado de la altiplanicie central, como Guanajuato, la casi totalidad de la población habla sólo español $(99.8 \%)$.

Además es digno de ser tomado en consideración el hecho de que esa vitalidad de la lengua maya en Yucatán no depende sólo de las razones cuantitativas aquí expuestas, sino también de circunstancias "cualitativas": el maya no es lengua hablada sólo por una mayoría de población rural o popular, marginada y carente de pres. tigio, sino que vive asimismo en boca de una buena parte de la población culta de la península. Goza, así, de un prestigio social de que carecen las demás lenguas indígenas de México ${ }^{14}$.

III. Para tratar de establecer la relación que pueda existir entre la articulación retrofleja de $/ \mathrm{r} /$ o $/ \bar{r} /$ y el maya, será conveniente atender a la capacidad lingüística de los informantes entrevistados por nosotros en las once localidades donde el fenómeno presentó alguna vitalidad digna de consideración ${ }^{15}$. Los datos sin-

frente a la castellana. En efecto, según el censo de 1950, los hablantes de maya en el estado de Yucatán representaban el $63 \%$ de la población (frente al $55.5 \%$ en 1970), y en Campeche, el 31.8\% (frente al 27\% en 1970). Pero resulta sorprendente - por no decir que sospechoso- que en Quintana Roo haya ido en aumento el número de hablantes de maya: el $43.7 \%$ en 1950 y el $54 \%$ en 1970. Cf. el cuadro estadístico que publica Alfonso Villa Rojas en su estudio sobre los "Patrones culturales mayas antiguos y modernos en las comunidades contemporáneas de Yucatán", incluido en Evon Z. Vost y Alberto Ruz (eds.), Desarrollo cultural de los mayas, México, 1971, pp. 353385. Temo que esa variación ascendente en el porcentaje de mayahablantes de Quintana Roo más refleje deficiencias en el proceso censal que la verdadera situación lingüística.

13 Porque, si bien en otros estados -como Oaxaca, por ejemplo- el número de hablantes de lenguas indigenas es también elevado, la situación presenta una fisonomía muy diferente, pues en ellos se hablan varias y muy diversas lenguas indoamericanas, frente a la unidad mayance peculiar de la península de Yucatán.

14 De los seis informantes cultos entrevistados en las localidades septen. trionales o centrales de la península yucateca (cf. nota 15), donde el maya ha conservado mayor vitalidad, cinco declararon hablar o conocer ampliamente la lengua indígena. El único que dijo desconocerla fue el informante de la capital, Mérida.

15 Es decir, en Tizimín, Valladolid, Mérida, Ticul, Felipe Carrillo Puerto, Campeche, Champotón, Chetumal, Mamantel, Emiliano Zapata y Huiman- 
tetizados en el cuadro que sigue son de imprecisa y discutible interpretación, y no creo que permitan llegar a conclusiones firmes.

ZONA NORTE

\begin{tabular}{|c|c|c|c|c|c|c|c|c|c|c|c|c|c|c|}
\hline & \multicolumn{2}{|c|}{ nzLMiN } & \multicolumn{2}{|c|}{ VALIADOLID } & \multicolumn{2}{|c|}{ MÉR IDA } & \multicolumn{2}{|c|}{ TICUL } & \multicolumn{2}{|c|}{ T. CARRILLO $\mathrm{T}$} & \multicolumn{2}{|c|}{ Г. CANTPECHE } & \multicolumn{2}{|c|}{ CHAMPOOÓN } \\
\hline & Maya & $\begin{array}{c}\text { No } \\
\text { maya }\end{array}$ & Maya & $\begin{array}{c}\text { No } \\
\text { maya }\end{array}$ & Maya & $\begin{array}{l}\text { No } \\
\text { maya }\end{array}$ & Maya & $\begin{array}{c}\text { No } \\
\text { maya }\end{array}$ & Maya & $\begin{array}{c}\text { No } \\
\text { maya }\end{array}$ & a Maya & $\begin{array}{c}\text { No } \\
\text { maya }\end{array}$ & Maya & $\begin{array}{l}\text { No } \\
\text { maya }\end{array}$ \\
\hline $\begin{array}{l}\text { Usan } \mathrm{R} \\
\text { No usan } \mathrm{R}\end{array}$ & 3 & ? & 3 & 1 & 1 & 2 & 3 & 9 & 4 & 1 & 2 & 2 & 1 & $\begin{array}{l}1 \\
4\end{array}$ \\
\hline
\end{tabular}

ZONA SUR

\begin{tabular}{|c|c|c|c|c|c|c|c|c|}
\hline & \multicolumn{2}{|c|}{ CHETUMAI. } & \multicolumn{2}{|c|}{ MAMANTEL } & \multicolumn{2}{|c|}{ E. ZAPATA } & \multicolumn{2}{|c|}{ HUIMANCUIHIO } \\
\hline & Maya & $\begin{array}{l}\text { No } \\
\text { maya }\end{array}$ & Maya & $\begin{array}{l}\text { No } \\
\text { maya }\end{array}$ & Maya & $\begin{array}{l}\text { No } \\
\text { Maya }\end{array}$ & Maya & $\begin{array}{l}\text { No } \\
\text { maya }\end{array}$ \\
\hline Usan R & & 5 & & 3 & & 3 & & 6 \\
\hline No usan $\mathrm{R}$ & & 2 & 1 & 3 & 1 & 3 & & 1 \\
\hline
\end{tabular}

En efecto, de los 69 informantes entrevistados en total, declararon conocer la lengua maya 28 (lo cual representa sólo su 40.5\%), en tanto que $41(59.5 \%)$ dijeron ser monolingües de castellano. De todos ellos, un total de $40(58 \%)$ articulaban la [R] con mayor o menor frecuencia, pero no la usaban los 29 restantes $(42 \%)$. Y -lo que podría ser más revelador- de los 40 usuarios de [R], 17 hablan maya ( $=42.5 \%$ o $24.6 \%$ del total absoluto), mientras que 23 ( $=57.5 \%$ o $33.3 \%$ respectivamente) desconocen la lengua indígena. $\mathrm{Y}$ de los 29 que no articulan la [R], $11(=38 \%$ o $16 \%$ del total absoluto) sí conocen el maya, pero $18(=62 \%$ o $26 \%$ respectivamente), no.

No creo que estos porcentajes permitan establecer una relación incuestionable entre el uso de la $[R]$ y el conocimiento del maya, por cuanto que la proporción de hablantes que se hallan en esa situación $(24.6 \%)$ es inferior a la de hablantes de maya que no se sirven de la [R] $(33.3 \%)$, si bien es cierto que esa inferioridad corresponde proporcionalmente al total de hablantes de maya $(40.5 \%)$ frente al de monolingües de castellano $(59.5 \%)$, con una insignificante diferencia - del $3 \%$ - en favor de la relación "hablante de maya / uso de [R]" ${ }^{16}$.

guillo. Las siete primeras localidades están situadas en la zona norte -propiamente peninsular- de la región estudiada, donde la lengua maya vive con vigor, en tanto que las cuatro últimas poblaciones pertenecen a la zona meridional, donde el número de hablantes de maya es ya muchísimo más reducido.

16 En efecto: si al $40.5 \%$ de hablantes de maya corresponde un $59.5 \%$ de monolingües castellanos, a un $\mathbf{2 4 . 6 \%}$ de maya-hablantes que usan [R] debería corresponder un $36.1 \%$ de monolingües castellanos que usaran [ $\mathrm{k}]$, en vez del $33.3 \%$ de nuestra estadística. Diferencia -repito- que considero insignificante y desprovista de todo significado. 
Lo que sí parece sorprendente es el hecho de que la proporción de usuarios de [R] sea tan alta en Chetumal y, sobre todo, en Huimanguillo ${ }^{17}$, donde nuestros informantes declararon desconocer la lengua indígena, lo cual parecería negar la relación entre el uso de la $[\mathrm{R}]$ y el conocimiento del maya. Pero en favor de esa hipótesis -que, por supuesto, y como después se verá, no trato de defender a ultranza- podría alegarse que un número tan reducido de informantes como los entrevistados en cada localidad no permite otorgarles una representatividad absoluta de la situación lingüistica de cada poblado. Además, es obvia la diferencia existente entre la situación lingüística de las siete poblaciones enclavadas en el norte de la península -en todas las cuales hemos hallado [R] en abundancia- y la de Chetumal y Huimanguillo, situadas en una región a que pertenecen otras localidades en las cuales la $[R]$ es muy débil $o$ inexistente y donde ya no se habla maya.

Dejando de lado - aunque sin desecharlas por completo- estas consideraciones de fría estadística, la situación lingüística general no parece permitir llegar a conclusiones seguras. Es cierto que la articulación retrofleja de $/ \mathrm{r} / \mathrm{o} / \overline{\mathrm{r}} /$ coincide, en líneas generales, con la región de adstrato maya, y que es particularmente frecuente en la zona septentrional, donde el idioma maya vive todavía con vigor. Pero no faltaría quien pensase en una coincidencia fortuita, y se negara a pasar por alto el especial caso de Huimanguillo.

Resulta, pues, oportuno traer a consideración un nuevo elemento de juicio, que contribuya a iluminar el panorama: el sistema fonético de la lengua maya.

IV. Los estudios más serios y sistemáticos hechos hasta ahora en torno al maya son obra -en su casi totalidad- de lingüistas antropólogos, cuya finalidad primordial ha sido el conocimiento y descripción estructural de la lengua maya, ya como sistema lingüistico general y con perspectiva histórica, ya como subsistemas dialectales hablados en la actualidad. Siempre, pues, se trata de hacer la descripción estructural de un sistema o subsistema lingüístico como tal. Pero no se ha pasado aún a hacer el análisis dialectal de cada uno de ellos. De ahí que en los estudios que he tenido la oportunidad de revisar se proporcione siempre el cuadro fonológico del sistema o subsistema objeto de estudio, pero no su cuadro fonético. Las observaciones o pormenores de carácter articulatorio o acústico son, en ellos, minoritarias, esporádicas o inexis-

17 Tan elevada como en Mérida, Felipe Carrillo Puerto o Valladolid. Aunque en Chetumal la mayor parte de los informantes usaban la [R], el índice de aparición de este alófono en su idiolecto era sumamente bajo, en tanto que en las poblaciones septentrionales de la península la frecuencia de su aparición es mucho más elevada. 
tentes ${ }^{18}$. De cualquier modo, esas monografías nos proporcionan algunas informaciones de sumo interés.

En primer lugar, la de que los fonemas vibrantes son extraños, escasos o mínimamente funcionales en las lenguas mayances, por cuanto que, históricamente, eran ajenos al sistema fonológico del maya clásico. $\mathrm{Ni} / \mathrm{r} / \mathrm{ni} / \overline{\mathrm{r}} /$ aparecen, efectivamente, en el cuadro de la fonología proto-mayance preparado por $T$. Kaufman ${ }^{19}$, ni en el sistema fonémico del proto-tzeltal-tzotzil reconstruido por el mismo autor ${ }^{20}$, ni tampoco en el del maya colonial organizado por $M$. Swadesh y sus colaboradores ${ }^{21}$.

El fonema $/ \mathrm{r} /$ puede hallarse en las lenguas mayances contemporáneas, pero - si no interpreto mal las informaciones que recojo de la bibliografía consultada- casi exclusivamente en préstamos de procedencia hispánica o como desarrollo del fonema maya $/ 1 /$. Fonema /r/ existe hoy en el maya del Petén, pero "sólo aparece en posición intervocálida: arux, duende" ${ }^{22}$; existe también en el tzeltal de Ocosingo, de Pinola y de San Bartolo ${ }^{23}$, y en otros dialectos tzeltales modernos ${ }^{24}$. Especialmente en voces de origen castellano, según García de León: "La aparición [de /r/] está restringida a préstamos del español; salvo el caso del término indígena contemporáneo /kerem/ 'muchacho' (que aparece como /kelem/

18 Por ningún motivo debe verse en estos comentarios el menor asomo de censura. Toda investigación debe estar orientada metodológicamente de acuerdo con los propósitos que la engendran. El conocimiento y posterior descripción de la lengua maya o de sus actuales variedades es, por supuesto, objetivo de primera magnitud, y una descripción estructural parece metodología adecuada a ese propósito. Que no se incluyan en ella pormenores fonéticos -aunque sea de lamentar para nuestro objetivo actual- no menoscaba en nada, naturalmente, el valor científico de la descripción.

19 Cf. Terrence S. Kaufman, "Materiales lingüísticos para el estudio de las relaciones internas y externas de la familia de idiomas mayanos", en F. Z. Vogt y A. Ruz, op. cit., pp. 81-136; véase, en especial, p. 84.

20 Cf. Terrence Kaufman, El proto-tzeltal-tzotzil. Fonologia comparada y diccionario reconstruido, México, 1972, p. 28.

21 Cf. Mauricio Swadesh, C. Álvarez y J. R. Bastarrachea, Diccionario de lementos del maya yucateco colonial, México, 1970, p. 11. Sólo en el cuadro de "Fonemas del protomaya" incluido por Norman A. McQuown en su estudio sobre "Los orígenes y la diferenciación de los mayas según se infiere del estudio comparativo de las lenguas mayanas" (en E. Z. Vost y A. Ruz, op. cit., p. 57), encuentro un fonema $/ \mathrm{r} /$, simple, germen del fonema /y/ de múltiples dialectos mayanos (p. 64).

22 Cf. Otto Schumann, Descripción estructural del maya itzá del Petén, Guatemala, México, 1971, pp. 27 y 29.

23 Cf Norman A. MCQuown, "Bilingüismo indígena y ladino: contrastes socioculturales", en N. A. McQuown y Julián PitT-Rivers (eds.), Ensayos de antropología en la zona central de Chiapas, México, 1970, pp. 625, 268 y 283.

24 Cf. Terrence Kaufman, Tzeltal-tzotzil comparative grammar, I: Phonology, University of Chicago, 1957, y El proto-tzeltal-tzotzil, cit. en nota 20. 
(quelem) en los siglos xvn y xviri)" ${ }^{25}$. La relación de la vibrante con la lateral en maya queda comprobada por el hecho -ampliamente documentado- de que los préstamos del castellano en que aparece $/ \mathrm{r} /$ suelen articularse en maya con / $/$ : tulesnáh 'durazno', teśeleśetík 'tijeras' ${ }^{26}$, naláś, nalaśaś 'naranjas' ${ }^{27}$. Y también por el hecho de que la articulación [r] sea considerada por los puristas mayanos como una forma "incorrecta" de $/ 1 /{ }^{28}$.

Por su parte, el fonema $/ \bar{r} /$ se mantiene todavía, al parecer, ajeno al sistema fonológico del maya. En efecto, aparece exclusivamente en voces de origen español ${ }^{29}$, y los indígenas lo pronuncian, por lo general, como [r] simple, inclusive los bilingües ${ }^{30}$. Tal vez resulte oportuno indicar que tanto esa $/ \overline{\mathrm{r}} /$, extraña al sistema fonológico maya, cuanto la $/ \mathrm{r} /$, ya más común en él, se articulan a veces como sordas ${ }^{31}$, cosa que resulta totalmente paralela a lo que sucede en varios dialectos mexicanos del español, en especial cuando se trata de los alófonos asibilados.

Para el problema que nos ocupa, la noticia de mayor interés podría ser la que proporciona Roberto Bruce sobre el sistema fonético de una lengua mayana que se ha conservado en el máximo aislamiento con respecto al español: el lacandón. Entre los fonemas de este idioma no existe ni $/ \mathrm{r} / \mathrm{ni}$ mucho menos $/ \overline{\mathrm{r}} /$, pero sí un sonido [r], alófono de $/ 1 /$, cuya articulación es precisamente retrofleja, como la realización del español yucateco que estamos estudiando: "La lateral $l$ tiene dos alófonos: $[l]$ y $[r]$. La $[l]$ es lateral,

25 Antonio García de León, Los elementos del tzotzil colonial y moderno, México, 1972, p. 20.-De procedencia castellana son, en el tzeltal moderno, los fonemas $/ \mathrm{r} / \mathrm{y} / \mathrm{d} /$, según Moisés Romero Cistillo, "Algunas observaciones sobre dialectologia tzeltal", en Anales del Instituto Nacional de Antropología e Historia, $13(1960), \mathrm{p}, 209$.

26 Aunque también asarón 'azadón'. Cf. Norman A. McQuown; Bilin. güismo, p. 278.

27 Pero, a la vez, narás, naranka: cf. Nicholas A. Hopkins, "Estudio preliminar de los dialectos del tzeltal y del tzotzil", en N. McQuown y J. PrrrRIVERs, Ensayos de antropologia, pp. 185-214; especialmente p. 213.

28 "La $r$ no figura en el alfabeto [maya] por dificultad de su pronunciación entre los indígenas; sólo el mestizo la pronuncia indebidamente diciendo HIRIXHOP, cerillo... cuando su pronunciación correcta es HILICHHOOP": SANTIAgo Pacheco Cruz, Compendio del idioma maya, 6a ed., Mérida, 1963, p. 4.

29 Cf. Terrence Kaufman, Tzeltal phonology and morphology, Berkeley, Los Ángeles, 1971 (UCPL, 61) , p. 14: "/ $/ \overline{\mathrm{r}} /$ occurs only in loans from Spanish".

30 Cf. N. McQuown, Bilingüismo, pp. 270-274 y 284: "una rana". Lo mismo en T. Kaurman, Tzeltal phonology, p. 13; “/ $/ \overline{\mathrm{r}} /$ is used only by acculturated speakers. It occurs initially and intervocalically only. Where acculturated speaker have $/ \overline{\mathrm{r}} /$, average and conservative speakers have $/ \mathrm{r} /$ ".

3 I Antonio García de León, Elementos del tzotzil, p. 20: "/r/... cuenta con un alófono sordo en posición final de silaba o palabra". Y T. Kaufman, Tzeltal phonology, p. 8: " $/ \overline{\mathrm{r}} /$. . usually voiced, optionally voiceless". 
alveolar y sonora. Ocurre únicamente en la posición inicial de palabra. $[r]$ es ligeramente retrofleja y sonora. Ocurre en las posiciones intermedia y final de palabra" y a veces se ensordece o debilita ${ }^{32}$. La inusitada atención a la fonética articulatoria con que Bruce describe estos sonidos del lacandón, tan distinta de la actitud básicamente fonologicista de los demás estudiosos del maya, permite ima. ginar que tal vez en otras lenguas mayances el sonido $[r]$ o el fonema /1/ pueda ser también "ligeramente retroflejo". Sólo encuentro otro testimonio que respalda esta suposición: el de O. Schumann, en su descripción fonética del fonema $/ 1 /$ según se articula en el idioma chol: la lateral $l$ tiene dos alófonos - uno sordo y otro sonoro- usados en distribución complementaria. "Ambos alófonos son ligeramente retroflejos en los hablantes monolingües" 33 .

V. En resumen:

1) La variante retrofleja [R] es particularmente usual en el español de la península yucateca, y su vitalidad disminuye, hasta desaparecer por completo, conforme nos alejamos de ese foco peninsular.

2) La región de $[R]$ coincide, a grandes trazos, con la zona de base lingüística maya, y el empleo de esa articulación retrofleja parece ser particularmente intenso en la zona donde la lengua maya subsiste con vigor. (Excepción importante es el caso de Huimanguillo, donde la articulación [R] se recogió -aunque con bajo índice de aparición- en boca de un elevado número de informantes, ninguno de los cuales hablaba maya).

3) La situación lingüística del núcleo mayoritario de los habitantes de Yucatán y Quintana Roo -donde el fenómeno estudiado presenta mayor vitalidad- es la de bilingüismo hispano-mayance.

4) La lengua maya conserva todavía hoy un relativamente alto prestigio en la zona yucateca, no sólo por el elevado número de sus usuarios, sino también por la importancia social de algunos de ellos.

5) La articulación de [R] retrofleja es propia tanto de bilingües hablantes de maya, como de monolingües en español.

6) El fonera $/ \mathrm{r} /$ parece ser ajeno al sistema fonológico del maya antiguo, y en los dialectos contemporáneos es poco frecuente. En ellos, la $/ r$ / suele aparecer en voces de origen español o ser un derivado moderno del fonema / / mayance.

7) En lacandón, la [r] es un simple alófono de $/ 1 /$, y su articulación es ligeramente retrofleja, como sucede con la $[R]$ del español yucateco. También el fonema $/ \mathrm{l} / \mathrm{del}$ chol es levemente retroflejo.

8) Otras variantes polimórficas de $/ \mathrm{r} / \mathrm{o} / \mathrm{r} /$ comunes en diversos dialectos del español mexicano -como las ensordecidas y quizá

32 Roberto D. Bruce, Gramática del lacandón, México, 1968, p. 24.

33 Otтo Schumann, La lengua chol de Tila, Chiapas, México, 1973, p. 11. 
las asibiladas- se encuentran tanto en hablantes yucatecos de español como en hablantes de maya.

VI. A la vista de todas estas circunstancias - un tanto imprecisas, a veces contradictorias, susceptibles siempre de diversa interpretación-, pienso que podría resultar un poco temerario atreverse a sostener una relación de causalidad entre la fonética de la lengua maya y la $[\mathbf{R}]$ del español yucateco. Varios de esos factores parecen apuntar hacia esta relación, en especial el caso de la articulación retrofleja de $[\mathrm{r}]</ 1 /$ en el lacandón, así como la coincidencia espacial entre el área del fenómeno fonético y la que corresponde actualmente al maya yucateco. Pero antes de inclinarse a proponer como segura esa relación, sería necesario conocer con exactitud cuáles son las particularidades fonéticas de la /r/ usada hoy en maya. $Y$, aun entonces, sería controvertib!e la relación supuesta, ya que -dado el caso de que la /r/ maya tuviera articulación levemente retrofleja en otras variedades además de la lacandona- cabría suponer que, siendo el fonema $/ \mathrm{r} /$ probablemente de origen español, el maya podría haberlo recibido ya con esa peculiar articulación que advertimos hoy en el español yucateco.

No obstante todas estas consideraciones que aconsejan cautela, pienso que la influencia del maya en la génesis de esa [R] retrofleja puede darse por cierta, si se piensa que su influjo ha podido ejercerse de manera indirecta.

Indicaba, en efecto, al comienzo de estas páginas que el habla yucateca - el español de la península de Yucatán- se nos presenta como un habla intensamente polimórfica. La [R] retrofleja es una de tantas muestras de ese acusado polimorfismo. Ahora bien, el polimorfismo -aunque represente el estado "normal", habitual, de todo sistema lingüístico ${ }^{34}$ - puede explicarse como el resultado inmediato de una "debilitación de la norma", según hace Menéndez Pidal en su colosal estudio sobre el antiguo romance castellano: "El lenguaje de ese período preliterario se nos muestra bullente de vida indómita y tumultuosa, con una variabilidad multicolor, aún más grande que la de los dialectos populares modernos. Parece que falta una norma cualquiera que rija el lenguaje vulgar antiguo" 35. O según hace, también, Manuel Alvar en su reciente estudio sobre el habla de Las Palmas: "El polimorfismo es consecuencia de una falta de nivelación en el sistema" 36 .

El polimorfismo particular de la península yucateca puede estar

34 Cf. mi estudio "En torno al polimorfismo" que se publicará en las Actas del $5^{\circ}$ Congreso de la A.I.H., celebrado en la Université de Bordeaux.

35 Ramón Menéndez Pidal, Origenes del español, $3^{a}$ ed., Madrid, 1950, \$ 107.

36 M. Alvar, Niveles socio-culturales en el habla de Las Palmas de Gran Canaria, Las Palmas, 1972, p. 163. Cf. también pp. 164-165. 
condicionado o haber sido favorecido por el estado de bilingüismo en que se encuentra la mayor parte de su población. De acuerdo con las precavidas consideraciones que hace Bertil Malmberg en torno a las situaciones que favorecen la interferencia lingüística -la influencia del sustrato o del adstrato ${ }^{3 i}-$, no debe sorprender en absoluto que la lengua indígena de Yucatán haya alcanzado a colorear el castellano hablado allí. Ya me he referido a la especial y prestigiosa situación de que goza el maya, frente a lo que sucede con las restantes lenguas amerindias, situación que favorece la interferencia ${ }^{38}$. Por otra parte, la articulación retrofleja [R] es -que sepamos- singular dentro de la lengua española, lo cual no permite encuadrarla dentro de las "tendencias estructurales" del español. Y son precisamente estos fenómenos insólitos los que Malmberg se inclina a aceptar como originados por las lenguas de adstrato ${ }^{3 n}$. "No hay -dice- influencia extranjera (interferencia, sustrato indígena) sino bajo condiciones particulares de mezcla de cultura y de tradiciones nacionales" (Tradición, p. 126). Y ésa es, precisamente, la situación histórico-cultural de Yucatán. No parece, pues, muy arriesgado atribuir - por lo menos en parte- al bilinguiismo de los yucatecos ese particular estado de "debilitación de la norma" que propicia el polimorfismo. $Y$ en este sentido si parece lícito suponer influencia de la lengua maya - por vía indirecta al menos- en la aparición de esa extraña realización retrofleja [R] dentro del español de Yucatán. No tiene por qué ser, necesariamente, una articulación de procedencia maya; pero su aparición en el español yucateco bien puede estar condicionada por la especial situación lingüística - bilingüe, de norma inestable, polimórfica- propia de ese territorio.

JuAN M. Lope Blanci

Universidad Nacional Autónoma de México.

37 Cf., en particular, L'espagnol dans le Nouvean Monde, I,und, 1948; "Liextension du castillan et le problème des substrats", en Actes du Colloque Internationale de Civilisations, Littíratures et Langues Romanes, Bucarest, 1959, pp. 249-260; y "Tradición hispánica e influencia indigena en la fonética hispanoamericana", en su libro Estudios de fonética hispánica, Madrid, 1965, Pp. 99-126.

ss Cf. B. Mambere, Tradición, p. 118: "El que haya influencia de sustrato o no en el encutentro de dos sistemas depende de su posición social y de los valores y del prestigio atribuidos a uno y otro por el grupo hablante".

39 "Si una tendencia enteramente nueva, aislada, aparece en un punto de la evolución... hay razones para buscar la explicación en alguna interferencia con otro sistema o en la influencia de hábitos fonéticos distintos (sustrato, superestratos, adstrato)": B. MALamberg, Tradición, p. 119. 